\title{
Cardiac manifestations of COVID-19 in Shenzhen, China
}

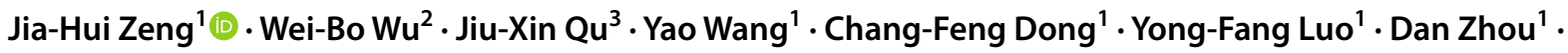 \\ Wen-Xia Feng ${ }^{1} \cdot$ Cheng Feng ${ }^{1}$
}

Received: 7 June 2020 / Accepted: 28 June 2020 / Published online: 28 July 2020

(c) Springer-Verlag GmbH Germany, part of Springer Nature 2020

\begin{abstract}
Purpose The coronavirus disease 2019 (COVID-19) outbreak has become a global public health concern; however, relatively few detailed reports of related cardiac injury are available. The aims of this study were to compare the clinical and echocardiographic characteristics of inpatients in the intensive-care unit (ICU) and non-ICU patients.

Methods We recruited 416 patients diagnosed with COVID-19 and divided them into two groups: ICU $(n=35)$ and non-ICU $(n=381)$. Medical histories, laboratory findings, and echocardiography data were compared.

Results The levels of myocardial injury markers in ICU vs non-ICU patients were as follows: troponin I $(0.029 \mathrm{ng} / \mathrm{mL}$ [0.007-0.063] vs $0.006 \mathrm{ng} / \mathrm{mL}$ [0.006-0.006]) and myoglobin $(65.45 \mu \mathrm{g} / \mathrm{L}$ [39.77-130.57] vs $37.00 \mu \mathrm{g} / \mathrm{L}$ [26.40-53.54]). Echocardiographic findings included ventricular wall thickening (12 [39\%] vs 1 [4\%]), pulmonary hypertension (9 [29\%] vs $0[0 \%])$, and reduced left-ventricular ejection fraction (5 [16\%] vs $0[0 \%])$. Overall, $10 \%$ of the ICU patients presented with right heart enlargement, thickened right-ventricular wall, decreased right heart function, and pericardial effusion. Cardiac complications were more common in ICU patients, including acute cardiac injury (21 [60\%] vs 13 [3\%]) (including 2 cases of fulminant myocarditis), atrial or ventricular tachyarrhythmia (3 [9\%] vs $3[1 \%])$, and acute heart failure (5 [14\%] vs $0[0 \%])$. Conclusion Myocardial injury marker elevation, ventricular wall thickening, pulmonary artery hypertension, and cardiac complications including acute myocardial injury, arrhythmia, and acute heart failure are more common in ICU patients with COVID-19. Cardiac injury in COVID-19 patients may be related more to the systemic response after infection rather than direct damage by coronavirus.
\end{abstract}

Keywords COVID-19 $\cdot$ Coronavirus $\cdot$ Myocarditis $\cdot$ Echocardiography $\cdot$ Intensive-care unit

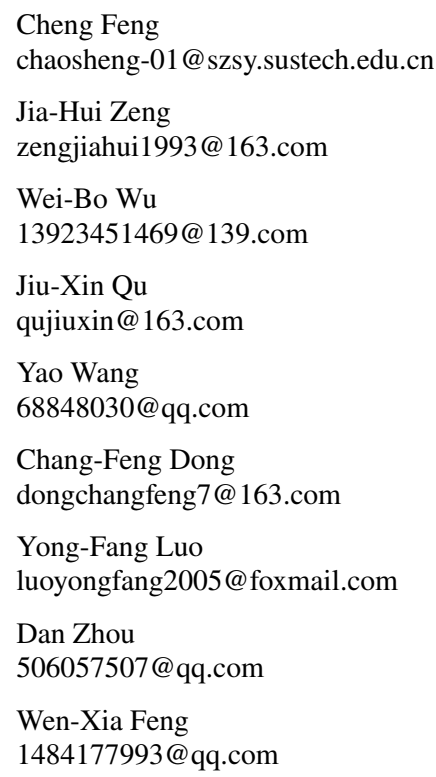

1 Department of Medical Ultrasonics, National Clinical Research Center for Infectious Disease, State Key Discipline of Infectious Disease, Shenzhen Third People's Hospital, Second Hospital Affiliated to Southern University of Science and Technology, NO. 29 Bulan Road, Shenzhen 518112, People's Republic of China

2 Department of Infectious Disease, National Clinical Research Center for Infectious Disease, State Key Discipline of Infectious Disease, Shenzhen Third People's Hospital, Second Hospital Affiliated to Southern University of Science and Technology, NO. 29 Bulan Road, Shenzhen 518112, People's Republic of China

3 Department of Clinical Laboratory, National Clinical Research Center for Infectious Disease, State Key Discipline of Infectious Disease, Shenzhen Third People's Hospital, Second Hospital Affiliated to Southern University of Science and Technology, NO. 29 Bulan Road, Shenzhen 518112, People's Republic of China 


\section{Background}

In December 2019, a group of cases of acute respiratory disease caused by severe acute respiratory syndrome coronavirus-2 (SARS-CoV-2), currently known as coronavirus disease 2019 (COVID-19), occurred in Wuhan, Hubei Province, China [1, 2]. At present, the numbers of cases and deaths are still rising, and the COVID-19 outbreak has become a global public health concern [3]. There have been reports about cardiac injury and complications including heart failure, arrhythmia, and myocardial infarction in COVID-19 patients, and troponin I (TnI) levels are higher in non-survivors $[4,5]$. However, information on differences in cardiac damage between severe and non-severe cases is relatively limited. The purpose of this case series is to describe and compare the clinical and echocardiographic characteristics of patients in the intensive-care unit (ICU) and non-ICU patients with COVID-19.

\section{Methods}

In this single-center retrospective study, we enrolled patients who were diagnosed with COVID-19 according to the WHO interim guidance from January 11 to April 1, 2020, at Shenzhen Third People's Hospital in China. A total of 416 patients were enrolled in this study and were divided into two groups: ICU and non-ICU patients. All the data have been shared with the WHO.

We acquired medical histories, laboratory findings, chest computed tomographic (CT) scans or X-rays, and echocardiography data from the patients' medical records. Laboratory confirmation of COVID-19 was conducted in two different institutions: the Chinese Center for Disease Control and Shenzhen Third People's Hospital. Other respiratory viruses, including respiratory syncytial virus, adenovirus, influenza A virus, influenza B virus, SARS coronavirus (SARS-CoV), MERS coronavirus (MERS-CoV), and parainfluenza virus, were also detected by RT-PCR.

We present continuous measurements as the mean \pm SD if they were normally distributed or as the median (IQR) if they were not normally distributed, and categorical variables are presented as the number (\%). When the data were normally distributed, independent-sample $t$ tests were used to compare the means of continuous variables; otherwise, the Mann-Whitney test was used. Nonnormally distributed repeated measurements were compared using a generalized linear mixed model. The Chi-square test was used to compare proportions of categorical variables. SPSS (Version 20.0) was used for all statistical analyses. In unadjusted comparisons, a two-sided $\alpha$ less than 0.05 was considered statistically significant.

\section{Results}

A total of 416 patients with COVID-19 were included in this study. Of these patients, 35 (8\%) were admitted and transferred to the ICU, because they required higher-level oxygen support measures or had multiple organ failure, and 381 (92\%) were admitted to isolation wards (Table 1).

Compared with patients who did not receive ICU care, patients who required ICU care were significantly older (64.00 years [59.50-68.00] vs 45.00 years [33.00-57.00]; $P<0.05)$ and were more likely to have underlying comorbidities, including hypertension (13 [37\%] vs 47 [12\%]), diabetes $(10[29 \%]$ vs $13[3 \%])$, arrhythmia (2 [6\%] vs $2[0.5 \%])$, and valvular disease (1 [3\%] vs $1[0.3 \%])$. The proportion of male patients in the ICU group was higher (23 [66\%] vs 175 [46\%]; $P<0.05)$. Patients in ICU had greater body mass index (BMI) and higher body temperature $(P<0.05)$. The arterial systolic pressure of the ICU patients was also higher than that of the non-ICU patients $(133.71 \pm 17.00 \mathrm{mmHg}$ vs $127.35 \pm 17.23 \mathrm{mmHg} ; \mathrm{P}<0.05)$. Heart rate did not differ between the two groups.

Numerous differences in laboratory findings were identified between the patients admitted to the ICU and those not admitted to the ICU (Table 2), including lower red blood cell and lymphocyte counts, higher white blood cell counts and fibrinogen, D-dimer, brain natriuretic peptide (BNP) and blood urea nitrogen levels, and longer prothrombin times in the ICU patients. Meanwhile, C-reactive protein, procalcitonin, and interleukin 6 (IL-6) levels were also higher in the ICU patients than in the non-ICU patients. Regarding myocardial injury markers, TnI and myoglobin (MYO) levels were significantly higher in the ICU patients, and the measurements for ICU vs non-ICU patients were as follows: $\mathrm{TnI}(0.029 \mathrm{ng} / \mathrm{mL}$ [0.007-0.063] vs $0.006 \mathrm{ng} /$ $\mathrm{mL}$ [0.006-0.006]) and MYO (65.45 $\mu \mathrm{g} / \mathrm{L}$ [39.77-130.57] vs $37.00 \mu \mathrm{g} / \mathrm{L}$ [26.40-53.54]). More patients presented with elevated TnI and MYO levels in the ICU group than in the non-ICU $(18 / 35$ [51\%] vs $11 / 310$ [4\%], $8 / 26$ [31\%] vs $22 / 148$ [15\%], respectively). In the ICU patients, the median partial pressure of oxygen $\left(\mathrm{PaO}_{2}\right)$ was $76.65 \mathrm{mmHg}$ (IQR, 59.10-96.60), and the median partial pressure of oxygen to the fraction of inspired oxygen ratio $\left(\mathrm{PaO}_{2} / \mathrm{FiO}_{2}\right)$ was 267.38 (IQR, 145.50-355.17), which were significantly lower than the values for the non-ICU patients. All patients' chest $\mathrm{X}$-rays and/or chest CT scans showed distributed patchy shadows or ground-glass opacities (Fig. 1).

Platelet counts, alanine aminotransferase, aspartate aminotransferase, creatinine, and creatine kinase-MB showed no significant differences between the two groups.

Due to considerations of reducing nosocomial infection and personnel shortages, we performed echocardiography and electrocardiography only for patients with severe 
Table 1 Baseline characteristics of COVID-19 patients

\begin{tabular}{lllr}
\hline Characteristic & ICU $(n=35)$ & Non-ICU $(n=381)$ & $P$ value $^{\mathrm{a}}$ \\
\hline Age, y & $64.00(59.50-68.00)$ & $45.00(33.00-57.00)$ & $<0.05$ \\
Body mass index & $24.63 \pm 3.31$ & $22.92 \pm 3.53$ & $<0.05$ \\
Sex & & & \\
Female & $12(34)$ & $206(54)$ & $<0.05$ \\
Male & $23(66)$ & $175(46)$ & \\
Comorbidities & & & $<0.05$ \\
Hypertension & $13(37)$ & $47(12)$ & 0.36 \\
Coronary artery disease & $2(6)$ & $11(3)$ & $<0.05$ \\
Arrhythmia & $2(6)$ & $2(0.5)$ & $<0.05$ \\
Valvular disease & $1(3)$ & $1(0.3)$ & $<0.05$ \\
Diabetes & $10(29)$ & $13(3)$ & 0.35 \\
COPD & $1(3)$ & $4(1)$ & 0.67 \\
Chronic kidney disease & $0(0)$ & $2(0.5)$ & 0.67 \\
Chronic liver disease & $0(0)$ & $2(0.5)$ & 0.39 \\
Tuberculosis & $0(0)$ & $8(2)$ & $<0.05$ \\
Highest temperature, ${ }^{\circ} \mathrm{C}$ & $38.50(38.00-39.20)$ & $38.00(37.70-38.50)$ & 0.13 \\
Heart rate, beats per minute & $96.794 \pm 13.7$ & $99.89 \pm 10.63$ & $<0.05$ \\
Arterial systolic pressure, $\mathrm{mmHg}$ & $133.71 \pm 17.00$ & $127.35 \pm 17.23$ & \\
\hline
\end{tabular}

Each value represents the median (IQR), the mean $\pm \mathrm{SD}$ or $n(\%)$

$C O P D$ chronic obstructive pulmonary disease

${ }^{\mathrm{a}} P<0.05$ was considered statistically significant disease, clinical symptoms, or a previous heart history (Table 3; Fig. 2). A total of 57 patients receive echocardiography, including 31 in ICU group and 26 in non-ICU group. The most common manifestations on echocardiography were a thickened interventricular septum dimension (IVSd) (12 [39\%] vs 1 [4\%]), a thickened left-ventricular posterior wall depth (LVPWd) (12 [39\%] vs 1 [4\%]), a reduced left-ventricular ejection fraction (LVEF) (5 [16\%] vs $0[0 \%])$, and pulmonary arterial hypertension $(9[29 \%]$ vs 0 [0\%]). Measurements of the IVSd, LVPWd, and pulmonary arterial systolic pressure (PASP) significantly differed between the groups, which were higher in the ICU group. The measurements for the ICU vs non-ICU patients were as follows: IVSd (11.00 mm [10.00-13.00] vs $10.00 \mathrm{~mm}$ [9.00-10.00]), LVPWd (11.00 mm [10.00-12.00] vs $10.00 \mathrm{~mm}[9.00-10.00])$, and PASP $(34.53 \pm 19.07 \mathrm{mmHg}$ vs $20.94 \pm 4.02 \mathrm{mmHg}$ ). The left atrium (LA) and left-ventricular diastolic diameter (LVDd) did not show significant differences between the two groups. Overall, $10 \%$ of the patients in the ICU presented with right heart enlargement, a thickened right-ventricular wall, decreased right heart function, and pericardial effusion. In addition, the measurements for left-ventricular diastolic function in the COVID19 patients in this study were normal.

The echocardiographic parameters of two patients who died in the ICU are shown in Fig. 3. Patient 1 was a 66-yearold male who required mechanical ventilation on the 9th day of hospitalization, underwent extracorporeal membrane oxygenation (ECMO) treatment on the 16th day, and died on the 35th day of hospitalization. Patient 2 was a 63-year-old male who required endotracheal intubation on the first day of hospitalization, was diagnosed with fulminant myocarditis, underwent ECMO treatment on the 8th day, and died on the $33^{\text {rd }}$ day of hospitalization. Both patients showed a thickened IVSd and an enlarged LVDd during treatment. Meanwhile, both patients had decreased LVEFs; however, the decreased LVEF in patient 1 appeared just before death, while that in patient 2 appeared early during hospitalization and gradually returned to normal after treatment. The PASP of the two patients gradually increased as the disease progressed; however, a sudden drop in pulmonary artery pressure occurred several days prior to death. Tricuspid annular plane systolic excursion (TAPSE) also showed a trend similar to that of PASP, with a sudden decline several days before death, suggesting impaired right cardiac function. The third patient died of multiple organ failure due to septic shock, leading to cardiac arrest.

As of April 1, 2020, $32(91 \%)$ and $381(100 \%)$ patients in the ICU and non-ICU groups recovered and were discharged, with median durations (from admission to discharge) of 38.00 days (IQR: $33.00-47.50$ ) and 20 days (IQR: 15.00-26.00), respectively (summarized in Table 4). Of these patients, 17 (49\%) ICU patients required non-invasive ventilation, and $18(51 \%)$ received invasive mechanical ventilation. Cardiac complications were more common in the ICU patients, including acute cardiac injury (21 [60\%] 
Table 2 Laboratory findings of COVID-19 patients on admission to the hospital

\begin{tabular}{|c|c|c|c|c|}
\hline Characteristic & Normal range & $\mathrm{ICU}(n=35)$ & Non-ICU $(n=381)$ & $P$ value $^{\mathrm{a}}$ \\
\hline Red blood cell count, $\times 10^{12} / \mathrm{L}$ & $4.0-5.5$ & $3.65(3.16-4.48)$ & $4.58(4.22-4.93)$ & $<0.05$ \\
\hline White blood cell count, $\times 10^{9} / \mathrm{L}$ & $3.5-9.5$ & $5.89(4.89-7.62)$ & $4.88(3.95-6.42)$ & $<0.05$ \\
\hline Lymphocyte percentage, $\%$ & $20-50$ & $0.75(0.45-1.05)$ & $1.35(1.04-1.82)$ & $<0.05$ \\
\hline Platelet count, $\times 10^{9} / \mathrm{L}$ & $125-350$ & $199.94 \pm 76.56$ & $213.18 \pm 76.38$ & 0.35 \\
\hline Prothrombin time, s & $11-15.1$ & $13.20(11.90-14.00)$ & $12.00(11.35-12.45)$ & $<0.05$ \\
\hline Fibrinogen, $\mathrm{g} / \mathrm{L}$ & $2-4$ & $4.55(3.60-5.99)$ & $3.79(3.13-4.62)$ & $<0.05$ \\
\hline D-dimer, $\mu \mathrm{g} / \mathrm{L}$ & $0-0.5$ & $1.25(0.62-3.02)$ & $0.36(0.24-0.54)$ & $<0.05$ \\
\hline Alanine aminotransferase, U/L & $9-50$ & $19.50(15.5-33.0)$ & $24.30(16.00-38.50)$ & 0.38 \\
\hline Aspartate aminotransferase, U/L & $15-40$ & $23.50(20.50-35.00)$ & $26.00(18.00-36.00)$ & 0.92 \\
\hline Blood urea nitrogen, $\mathrm{mmol} / \mathrm{L}$ & $2.8-7.6$ & $4.94(4.26-7.74)$ & $4.38(3.50-5.91)$ & $<0.05$ \\
\hline Creatinine, $\mu \mathrm{mol} / \mathrm{L}$ & $64-104$ & $69.00(57.5-99.50)$ & $69.00(56.00-79.50)$ & 0.37 \\
\hline Troponin I, ng/mL & $<0.026$ & $0.029(0.007-0.063)$ & $0.006(0.006-0.006)$ & $<0.05$ \\
\hline Myoglobin, $\mu \mathrm{g} / \mathrm{L}$ & $<100$ & $65.45(39.77-130.57)$ & $37.00(26.40-53.54)$ & $<0.05$ \\
\hline Troponin I elevated & - & $18 / 35(51)$ & $11 / 310(4)$ & $<0.05$ \\
\hline Myoglobin elevated & - & $8 / 26(31)$ & $22 / 148(15)$ & $<0.05$ \\
\hline BNP, pg/L & $<100$ & $19.69(6.67-33.29)$ & $1.94(0.95-3.80)$ & $<0.05$ \\
\hline Creatine kinase-MB, U/L & $<25$ & $1.20(0.77-1.82)$ & $0.88(0.55-1.41)$ & 0.16 \\
\hline C-reactive protein, $\mathrm{mg} / \mathrm{L}$ & $0.8-8$ & $55.30(11.32-124.53)$ & $23.61(6.64-66.80)$ & $<0.05$ \\
\hline Procalcitonin, $\mathrm{ng} / \mathrm{mL}$ & $<0.05$ & $0.21(0.16-0.26)$ & $0.22(0.18-0.28)$ & $<0.05$ \\
\hline Interleukin $6, \mathrm{pg} / \mathrm{ml}$ & $<7$ & $46.18(27.39-150.40)$ & $15.07(5.22-43.07)$ & $<0.05$ \\
\hline $\mathrm{PH}$ & $7.35-7.45$ & $7.45(7.43-7.47)$ & $7.42(7.40-7.44)$ & $<0.05$ \\
\hline $\mathrm{PaCO}_{2}, \mathrm{mmHg}$ & $35-48$ & $37.45(32.80-40.00)$ & $40.00(37.80-42.80)$ & $<0.05$ \\
\hline $\mathrm{PaO}_{2}, \mathrm{mmHg}$ & $83-108$ & 76.65 (59.10-96.60) & $94.25(82.90-108.00)$ & $<0.05$ \\
\hline $\mathrm{PaO}_{2} / \mathrm{FIO}_{2}$ & $400-500$ & $267.38(145.50-355.17)$ & $426.19(362.16-485.71)$ & $<0.05$ \\
\hline
\end{tabular}

Each value represents the median (IQR), the mean $\pm \mathrm{SD}$ or $n / N(\%)$

$M B$ muscle and brain type, $B N P$ brain natriuretic peptide, $\mathrm{COVID}-19$ coronavirus disease $2019, \mathrm{FIO}_{2}$ fraction of inspired oxygen, $\mathrm{PaCO} \mathrm{O}_{2}$ partial pressure of carbon dioxide, $\mathrm{PaO}_{2}$ partial pressure of oxygen

${ }^{\mathrm{a} P}<0.05$ was considered statistically significant

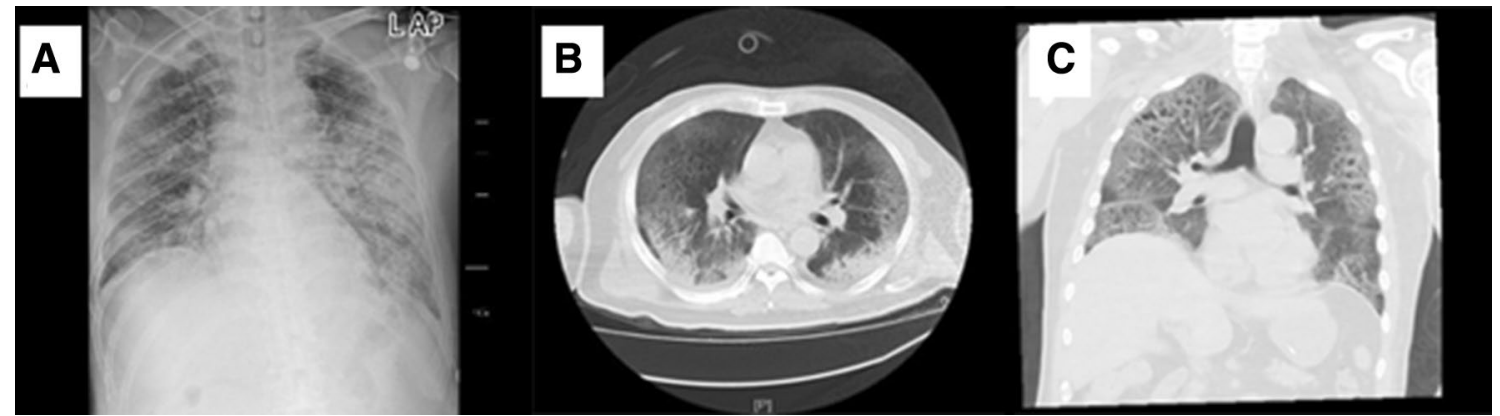

Fig. 1 The chest imaging of patient 2. a Bedside chest radiograph on the first day of admission. $\mathbf{b}$, $\mathbf{c}$ Computerized tomography on the 9th day showed ground-glass changes typical of viral pneumonia

vs 13 [3\%]) (including 2 cases of fulminant myocarditis), atrial or ventricular tachyarrhythmia (3 [9\%] vs $3[1 \%])$, and acute heart failure (5 [14] vs 0 [0\%]). Electrocardiograph abnormalities mainly include atrial or ventricular tachycardia, $\mathrm{T}$ wave changes, atrial flutter, bundle branch block, etc. Acute cardiac injury was defined as blood levels of hypersensitive TnI above the 99th percentile upper reference limit $(>0.028 \mathrm{ng} / \mathrm{mL})$ or new abnormalities shown on electrocardiography and echocardiography. Acute myocardial infarction did not differ between the two groups. In the non-ICU group, five patients received non-invasive ventilation support. Five (15\%) ICU patients required ECMO, and 
Table 3 Echocardiographic characteristics of 57 COVID-19 patients

\begin{tabular}{lllr}
\hline Echocardiographic findings & ICU $(n=31)$ & Non-ICU $(n=26)$ & $P$ value $^{\mathrm{a}}$ \\
\hline LA, mm & $31.00 \pm 3.04$ & $31.16 \pm 2.87$ & 0.86 \\
LA $>35 \mathrm{~mm}$ & $1(3)$ & $1(4)$ & 0.90 \\
LVDd, mm & $46.35 \pm 4.58$ & $45.81 \pm 4.37$ & 0.67 \\
LVDd $>55 \mathrm{~mm}$ & $2(6)$ & $1(4)$ & 0.66 \\
IVSd, mm & $11.00(10.00-13.00)$ & $10.00(9.00-10.00)$ & $<0.05$ \\
IVSd $>11 \mathrm{~mm}$ & $12(39)$ & $1(4)$ & $<0.05$ \\
LVPWd, mm & $11.00(10.00-12.00)$ & $10.00(9.00-10.00)$ & $<0.05$ \\
LVPWd $>11$ mm & $12(39)$ & $1(4)$ & $<0.05$ \\
LVEF, \% & $63.00(59.00-66.00)$ & $63.50(60.00-67.00)$ & 0.43 \\
LVEF $<55 \%$ & $5(16)$ & $0(0)$ & $<0.05$ \\
PASP, mmHg & $34.53 \pm 19.07$ & $20.94 \pm 4.02$ & $<0.05$ \\
PASP $>40$ mmHg & $9(29)$ & $0(0)$ & $<0.05$ \\
Right heart enlargement & $3(10)$ & $0(0)$ & 0.10 \\
Thickened right-ventricular wall & $3(10)$ & $0(0)$ & 0.10 \\
Decreased right heart function & $3(10)$ & $0(0)$ & 0.10 \\
Pericardial effusion & $3(10)$ & $0(0)$ & 0.10 \\
\hline
\end{tabular}

Each value represents the median (IQR), the mean \pm SD or $n(\%)$. Right heart enlargement was defined as right atrial and/or ventricular enlargement; a thickened right-ventricular wall was defined as right-ventricular anterior wall thickness $>5 \mathrm{~mm}$; decreased right heart function was defined as tricuspid annular plane systolic excursion $<16 \mathrm{~mm}$, a right-ventricular fractional area change $<35 \%$ and/or Systolic S' of the anterior tricuspid annulus with tissue doppler $<10 \mathrm{~cm} / \mathrm{s}$

$L A$ left atrium, $L V D d$ left-ventricular diastolic diameter, IVSd interventricular septum dimension, $L V P W d$ left-ventricular posterior wall depth, $L V E F$ left-ventricular ejection fraction, $P A S P$ pulmonary arterial systolic pressure

${ }^{a} P<0.05$ was considered statistically significant

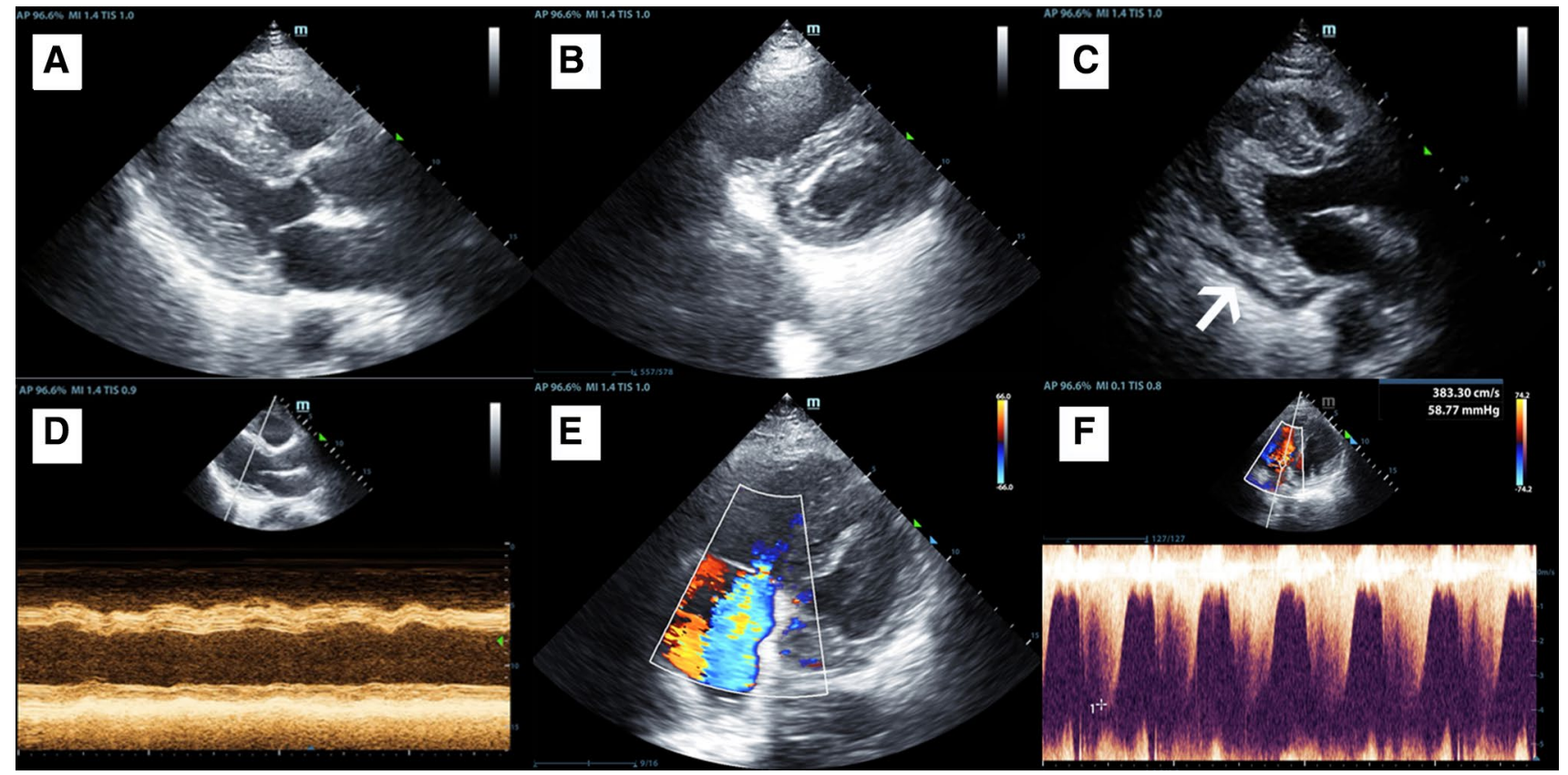

Fig. 2 Echocardiographic images of patients with COVID-19. a Diffuse thickening of the left-ventricular wall. b Right-ventricular enlargement and left-ventricular wall thickening. c A small amount of pericardial effusion behind the left-ventricular posterior wall (white arrow). d M-mode graph of the left-ventricular basement showed ventricular wall dyskinesia and a decreased left-ventricular ejection fraction. e Color Doppler showed moderate-to-severe tricuspid regurgitation. f Continuous wave Doppler showed pulmonary hypertension 


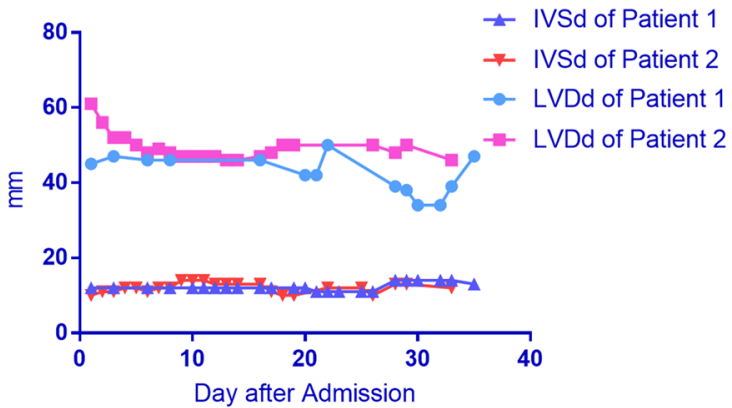

A

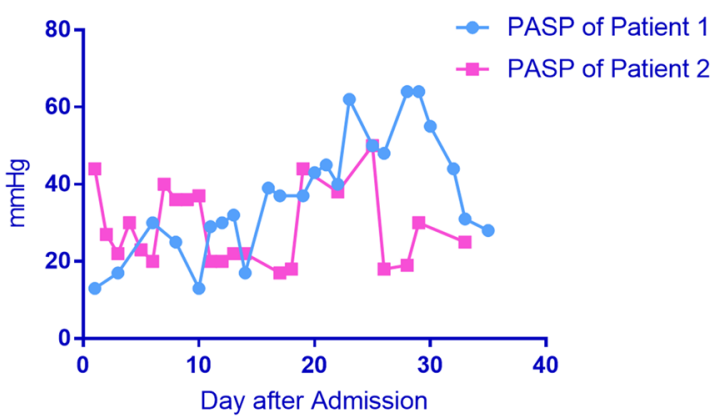

C

Fig. 3 Echocardiographic parameters of two non-survivors. Patient 1 died on the 35th day of hospitalization, and patient 2 died on the 33rd day of hospitalization. a Both patients showed a thickened IVSd and an enlarged LVDd during treatment. b Both patients had decreased LVEFs; however, the decreased LVEF in patient 1 appeared just before death, while that in patient 2 appeared early during hospitalization and gradually returned to normal after treatment. c The

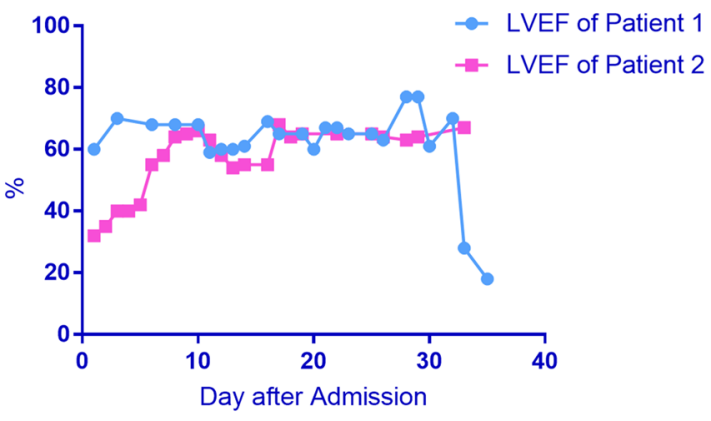

B

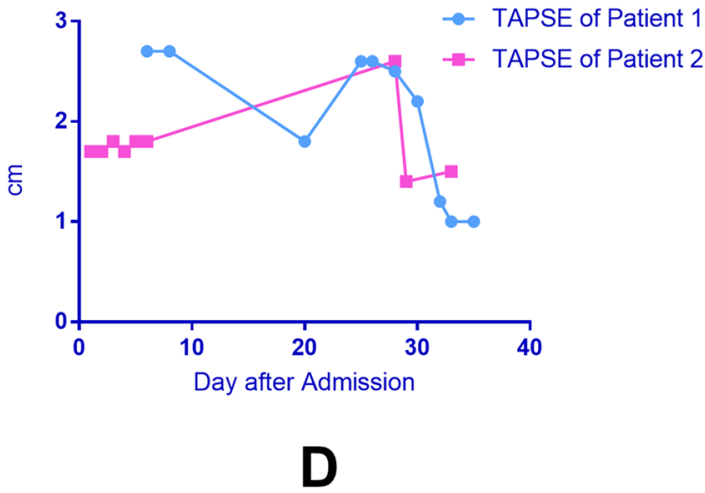

PASP of the two patients gradually increased as the disease progressed; however, a sudden drop occurred several days prior to death. d TAPSE also showed a trend similar to that of PASP, with a sudden decline several days before death. $L V D d$ left-ventricular diastolic diameter, IVSd interventricular septum dimension, $L V E F$ left-ventricular ejection fraction, $P A S P$ pulmonary arterial systolic pressure, TAPSE tricuspid annular plane systolic excursion
Table 4 Treatments and outcomes of COVID-19 patients

\begin{tabular}{llll}
\hline & ICU $(n=35)$ & Non-ICU $(n=381)$ & $P$ value $^{\mathrm{a}}$ \\
\hline Complications & & & \\
Acute cardiac injury & & $13(3)$ & $<0.05$ \\
Atrial or ventricular tachyarrhythmia & $3(9)$ & $3(1)$ & $<0.05$ \\
Acute myocardial infarction & $1(3)$ & $1(0.3)$ & 0.16 \\
Acute heart failure & $5(14)$ & $0(0)$ & $<0.05$ \\
Oxygen support & & $5(1)$ & $<0.05$ \\
Non-invasive ventilation & $17(49)$ & $0(0)$ & $<0.05$ \\
Invasive mechanical ventilation & $18(51)$ & $0(0)$ & $<0.05$ \\
ECMO & $5(15)$ & & \\
Prognosis & & $381(100)$ & $<0.05$ \\
Discharge & $32(91)$ & $0(0)$ & - \\
Death & $3(9)$ & $20.00(15.00-26.00)$ & \\
Days from admission to discharge & $38.00(33.00-47.50)$ & - & \\
Days from admission to death & $31.33 \pm 5.03$ & &
\end{tabular}

Each value represents the median (IQR), the mean \pm SD or $n(\%)$

ECMO extracorporeal membrane oxygenation

${ }^{\text {a }} P<0.05$ was considered statistically significant

${ }^{b}$ Defined as blood levels of troponin I above the 99th percentile upper reference limit $(>0.028 \mathrm{ng} / \mathrm{mL})$ or new abnormalities shown on electrocardiography and echocardiography 
three of these patients died during hospitalization; the mean time from admission to death was $31.33 \pm 5.03$ days.

Of the 416 patients in this study, 18 had a previous history of heart disease, mainly including coronary heart disease, arrhythmia, and valvular disease, and 398 had no heart disease history. We compared cardiac markers, echocardiography findings, and complications between these two types of patients (Table 5). The results showed that patients with a previous history of heart disease were more likely to have elevated TnI (5/14 [36\%]), acute myocardial injury (6/18 [33\%]), atrial or ventricular arrhythmia (3/18 [17\%]), and acute myocardial infarction (2/18 [11\%]). No significant difference in the echocardiography measurements of the two groups was identified.

\section{Discussion}

COVID-19 patients in the ICU were older and had a higher $\mathrm{BMI}$ and more comorbidities, such as a previous history of diabetes, hypertension, arrhythmia, and valvular disease, than those who were not admitted to the ICU. The arterial
Table 5 Characteristics of patients with or without previous heart disease

\begin{tabular}{|c|c|c|c|}
\hline & $\begin{array}{l}\text { Previous heart disease } \\
\text { present }(n=18)\end{array}$ & $\begin{array}{l}\text { Previous heart disease } \\
\text { absent }(n=398)\end{array}$ & $P$ value $^{\mathrm{a}}$ \\
\hline \multicolumn{4}{|l|}{ Laboratory } \\
\hline Troponin I, ng/mL & $0.020(0.006-0.033)$ & $0.006(0.006-0.006)$ & $<0.05$ \\
\hline Myoglobin, $\mu \mathrm{g} / \mathrm{L}$ & $44.77(35.70-100.55)$ & $38.63(27.03-63.30)$ & 0.18 \\
\hline Troponin I elevated & $5 / 14(36)$ & $24 / 331(7)$ & $<0.05$ \\
\hline Myoglobin elevated & $3 / 11(27)$ & $27 / 163(17)$ & 0.41 \\
\hline \multicolumn{4}{|l|}{ Echocardiography } \\
\hline $\mathrm{LA}, \mathrm{mm}$ & $32.33 \pm 2.87$ & $30.76 \pm 2.91$ & 0.15 \\
\hline $\mathrm{LA}>35 \mathrm{~mm}$ & $1 / 10(10)$ & $1 / 47(2)$ & 0.32 \\
\hline LVDd, mm & $45.00(45.00-46.00)$ & $46.00(43.00-48.00)$ & 0.95 \\
\hline LVDd $>55 \mathrm{~mm}$ & $0 / 10(0)$ & $3 / 47(6)$ & 0.41 \\
\hline IVSd, mm & $11.00(10.00-11.00)$ & $10.00(10.00-12.00)$ & 0.55 \\
\hline IVSd $>11 \mathrm{~mm}$ & $2 / 10(20)$ & $11 / 47(23)$ & 0.82 \\
\hline LVPWd, mm & $10.00(10.00-11.00)$ & $10.00(9.00-12.00)$ & 0.35 \\
\hline LVPWd >11 mm & $2 / 10(20)$ & $11 / 47(23)$ & 0.82 \\
\hline LVEF, \% & $60.00(58.00-63.00)$ & $64.00(60.00-67.00)$ & 0.06 \\
\hline LVEF $<55 \%$ & $1 / 10(10)$ & 4/47 (9) & 0.88 \\
\hline PASP, $\mathrm{mmHg}$ & $20.00(18.00-23.00)$ & $25.00(20.00-31.00)$ & 0.18 \\
\hline PASP $>40 \mathrm{mmHg}$ & $1 / 10(10)$ & $8 / 47(17)$ & 0.58 \\
\hline Right heart enlargement & $1 / 10(10)$ & $2 / 47(4)$ & 0.45 \\
\hline Thickened right-ventricular wall & $0 / 10(0)$ & $3 / 47(6)$ & 0.41 \\
\hline Decreased right heart function & $1 / 10(10)$ & $2 / 47(4)$ & 0.45 \\
\hline Pericardial effusion & $0 / 10(0)$ & $3 / 47(6)$ & 0.55 \\
\hline \multicolumn{4}{|l|}{ Complications } \\
\hline Acute cardiac injury ${ }^{\mathrm{b}}$ & $6 / 18(33)$ & $28 / 398(7)$ & $<0.05$ \\
\hline Atrial or ventricular tachyarrhythmia & $3 / 18(17)$ & $3 / 398(0.8)$ & $<0.05$ \\
\hline Acute myocardial infarction & $2 / 18(11)$ & 0/398 (0) & $<0.05$ \\
\hline Acute heart failure & $1 / 18(6)$ & $4 / 398(1)$ & 0.20 \\
\hline
\end{tabular}

Each value represents the median (IQR), the mean \pm SD or $n / N(\%)$. Troponin I elevated was defined as troponin I $>0.026 \mathrm{ng} / \mathrm{mL}$. Myoglobin elevated was defined as myoglobin $>100 \mu \mathrm{g} / \mathrm{L}$. Right heart enlargement was defined as right atrial and/or ventricular enlargement; A thickened right-ventricular wall was defined as right-ventricular anterior wall thickness $>5 \mathrm{~mm}$; decreased right heart function was defined as tricuspid annular plane systolic excursion $<16 \mathrm{~mm}$, a right-ventricular fractional area change $<35 \%$ and/or Systolic S' of the anterior tricuspid annulus with tissue Doppler $<10 \mathrm{~cm} / \mathrm{s}$

$L A$ left atrium, $L V D d$ left-ventricular diastolic diameter, IVSd interventricular septum dimension, $L V P W d$ left-ventricular posterior wall depth, $L V E F$ left-ventricular ejection fraction, PASP pulmonary arterial systolic pressure

${ }^{a} P<0.05$ was considered statistically significant

${ }^{b}$ Defined as blood levels of troponin I above the $99^{\text {th }}$ percentile upper reference limit $(>0.028 \mathrm{ng} / \mathrm{mL})$ or new abnormalities shown on electrocardiography and echocardiography. 
systolic pressure of the ICU patients was also higher, suggesting that age, BMI, and comorbidities may be risk factors for a poor outcome due to the weak immune function of these patients $[6,7]$. Studies have shown that hypertension and diabetes can lead to a decrease in organ reserves [8,9]. This phenomenon suggests that in patients with hypertension and diabetes, organ reserves are reduced, thus reducing the ability to withstand viral attacks and leading to more severe symptoms requiring ICU treatment. In the 416 patients with COVID-19 in this study, we did not observe a greater number of men than women, which is different from the results of a previous study [4]. However, the proportion of males was found to be higher in the ICU group, which was consistent with the reports of MERS-CoV and SARS-CoV infection $[6,10]$. The lower female susceptibility to viral infection may be related to protection conferred by the $\mathrm{X}$ chromosome and sex hormones, which may play important roles in innate and adaptive immunity [7]. Another possible cause is sympathetic hyperactivity. Damage to norepinephrine reuptake in the elderly population increases the sympathetic signal, especially the cardiac sympathetic signal, causing greater cardiac stimulation [11]. Whether the difference in the neurobiology of sympathetic nerves based on age and sex renders COVID-19 more likely to cause heart damage in elderly patients and patients of a specific sex is unknown.

Compared with the non-ICU patients, the ICU patients had more laboratory abnormalities. The most common laboratory abnormalities in this study were a decreased lymphocyte count, a prolonged prothrombin time, and elevated levels of blood urea nitrogen, BNP, C-reactive protein, procalcitonin, D-dimer, and IL-6. These abnormalities suggest that SARS-CoV-2 infection may be related to cellular immune deficiency, coagulation activation, and injury to multiple organs. Moreover, $\mathrm{PaO}_{2}$ and $\mathrm{PaO}_{2} / \mathrm{FiO}_{2}$ were significantly lower in the ICU group, suggesting more serious hypoxia in these patients. Activated macrophages can release IL-6 and other cytokines and promote the expression of adhesion molecules. Dysfunctional endothelial cells become adhesive and secrete procoagulant factors [12]. These changes further accelerate the inflammation of blood vessels and enhance the prethrombotic state. The presence of microvascular lesions and microthrombi can also cause patients to be prone to multiple organ failure, further aggravating the state of heart damage and heart failure. Therefore, patients with elevated IL-6 and D-dimer must be monitored closely to prevent micro-embolism.

Regarding myocardial injury markers, TnI and MYO levels were significantly higher in the ICU patients. Overall, $49 \%$ of the patients in ICU group and $8 \%$ of the patients in non-ICU group had elevated levels of TnI. A recent report showed that among 41 COVID-19 patients diagnosed in Wuhan, 5 (12\%) were diagnosed with virus-related heart injuries, and 4 of the 5 patients were admitted to the ICU, accounting for $31 \%$ of the total number of ICU patients [5]. Recent research shows that $\mathrm{TnI}$ and BNP peaked within 1 week before COVID-19 patients died and indicates that elevated cardiac markers may be a warning sign of a poor prognosis [13]. The increase or the dynamic increase in TnI during hospitalization can increase the risks of requiring ventilation support, arrhythmia, and mortality by five times [14]. In the present study, cardiac complications were more common in the ICU group than in the non-ICU, including acute cardiac injury, atrial or ventricular tachyarrhythmia, and acute heart failure. These results suggest that when patients are infected with SARS-CoV-2, the incidence of cardiovascular system diseases may increase due to the systemic inflammatory response and immune system disorders, and may be associated with exacerbation of the disease and the need for treatment in the ICU.

Considering the need for isolation and the inconvenience of examining critically ill patients, echocardiography is the main means of observing and monitoring structural and functional abnormalities of the heart in patients with COVID-19. In this study, the most common manifestation on echocardiography was left-ventricular thickening, followed by a reduced LVEF and pulmonary arterial hypertension. Pathological examinations showed that interstitial mononuclear inflammatory infiltrates, dominated by lymphocytes in the lung tissues, suggest inflammatory injury [15]. Pulmonary embolism, small vessel spasms, and subsequent pulmonary fibrosis can also lead to pulmonary hypertension [16]. Recent pulmonary CT angiography findings in patients with COVID-19 also confirm the presence of pulmonary microembolism [17]. In the present study, proximal pulmonary embolism, and right-ventricular outflow tract stenosis and pulmonary valve stenosis were excluded in some patients by ultrasound or CT examination, which may exclude the pulmonary hypertension caused by the above reasons.

Hypoxia and the accompanying metabolic disorders can lead to pulmonary vasospasm, inflammatory damage, and hypercapnia, followed by transient pulmonary hypertension, thus affecting right heart function. Myocardial cell ischemia and hypoxia can impact the heart rhythm and left heart function [18]. Some studies [19] have shown that intermittent or continuous severe hypoxia can lead to an earlier and greater degree of inflammatory response and cell damage in the heart. A previous study suggested that hypoxia can regulate morphological changes and remodeling of the myocardium and can present as wall thickening [20], suggesting that the thickening of the cardiac wall observed in the patients in this study may not only be the result of the immune response, but that hypoxia may also cause changes in ventricular morphology. Fewer patients presented with right heart enlargement, a thickened rightventricular wall, and decreased right heart function. However, according to our clinical observations, the occurrence 
of the above conditions often indicates that the pulmonary lesion is aggravated or the course of disease is entering the late stage. In general, fibrosis is irreversible, and if pulmonary hypertension suddenly decreases, right heart function may be decreased. A few patients had pericardial effusion and left atrial or ventricular enlargement, which may be related to the severity of myocardial inflammation.

The pathogenesis of acute myocardial injury related to virus infection is not currently clear; however, several widely recognized possible mechanisms have been suggested. First, direct myocardial injury caused by viral replication may play a role. Second, SARS-CoV-2 has been suggested to infect cells through the ACE2 receptor, which is widely expressed in the cardiovascular system [21]. However, a recent pathological report showed that no obvious intranuclear or cytoplasmic viral inclusions were identified in the heart tissue [15]. Another report also shows that COVID-19 patients have increased level of interleukin, indicating that the T-cell reaction is enhanced [5]. In our study, we also found a significant increase in IL-6 in blood samples from COVID-19 patients, which was significantly greater in the ICU patients than in the non-ICU patients. These results all suggest that the replication of the virus in the myocardium is unclear, and that severe immune injury may play a key role in the pathogenesis of viral myocarditis [22, 23].

The results of this study show that patients with a previous history of heart disease are more likely to have cardiac complications, as demonstrated by the higher proportion of patients with a previous history of heart disease in the ICU group than in the non-ICU group, and have similar characteristics in terms of cardiac complications, which suggests that patients with a previous cardiac history may be more serious after being infected with SARS-CoV-2 and more likely to need ICU treatment. In this study, cardiac history had no significant effect on the echocardiographic measurements, suggesting that the echocardiographic differences between two groups were related more to the virus infection. While the pathophysiological mechanism of cardiac manifestation in COVID-19 patients remains uncertain, the detection of cardiac damage in such patients may help to identify patients at greater risk of complications.

This study has several limitations. Among the 416 patients, not all patients received echocardiography to characterize cardiac injury. COVID-19 patients may present with reduced strain or impaired perfusion on echocardiography. In addition, the study failed to detect the blood catecholamine of the patients and was unable to confirm the relationship between sympathetic overactivation and myocardial injury, which needs further study.

Acknowledgements We would like to thank the staff members at the China CDC for their contributions and assistance in the diagnosis of patients.
Author contributions $\mathrm{JHZ}$ and CF had full access to all of the data in the study and assume responsibility for the integrity and accuracy of the data. Concept, design, and supervision: CF. Acquisition, analysis, or interpretation of the data: all authors. Drafting of the manuscript: JHZ. Critical revision of the manuscript for important intellectual content: WBW.

Funding This manuscript is funded by Guangdong Medical Research Fund (Grant B2019163).

\section{Compliance with ethical standards}

Conflict of interest On behalf of all authors, the corresponding author states that no conflicts of interest exist.

Ethics approval The study was approved by the Ethics Committee of Shenzhen Third People's Hospital (No. 2020-078).

Consent to participate and publication Written informed consent was obtained from the patients. A copy of the written consent is available for review by the Editor of this journal.

Availability of data and material All available information is contained within the present manuscript.

\section{References}

1. Lu H, Stratton CW, Tang YW. Outbreak of pneumonia of unknown etiology in Wuhan, China: the mystery and the miracle. J Med Virol. 2020. https://doi.org/10.1002/jmv.25678.

2. Hui DS, Azhar EI, Madani TA, Ntoumi F, Kock R, Dar O, et al. The continuing 2019-nCoV epidemic threat of novel coronaviruses to global health-the latest 2019 novel coronavirus outbreak in Wuhan, China. Int J Infect Dis. 2020;91:264-6. https:// doi.org/10.1016/j.jijid.2020.01.009.

3. WHO. Coronavirus disease 2019 (COVID-19) situation report-77. 2020. https://www.who.int/docs/default-source/coron aviruse/situation-reports/20200406-sitrep-77-covid-19.pdf?sfvrs $\mathrm{n}=21 \mathrm{~d} 1 \mathrm{e} 632 \_2$. Accessed April 6, 2020.

4. Chen N, Zhou M, Dong X, Qu J, Gong F, Han Y, et al. Epidemiological and clinical characteristics of 99 cases of 2019 novel coronavirus pneumonia in Wuhan, China: a descriptive study. Lancet. 2020. https://doi.org/10.1016/S0140-6736(20)30211-7.

5. Huang C, Wang Y, Li X, Ren L, Zhao J, Hu Y, et al. Clinical features of patients infected with 2019 novel coronavirus in Wuhan, China. Lancet. 2020. https://doi.org/10.1016/S0140 -6736(20)30183-5.

6. Channappanavar R, Fett C, Mack M, Ten Eyck PP, Meyerholz DK, Perlman S. Sex-based differences in susceptibility to severe acute respiratory syndrome coronavirus infection. J Immunol. 2017;198:4046-53. https://doi.org/10.4049/jimmunol.1601896.

7. Jaillon S, Berthenet $K$, Garlanda C. Sexual dimorphism in innate immunity. Clin Rev Allergy Immunol. 2019;56:308-21. https:// doi.org/10.1007/s12016-017-8648-x.

8. Hadadi L, Serban RC, Juhasz T, Tepes O, Somkereki C, Demjen $\mathrm{Z}$, et al. Renal dysfunction is associated with lower incidence of positive fractional flow reserve values in patients with severe hypertension. Minerva Cardioangiol. 2020. https://doi. org/10.23736/S0026-4725.20.05103-8.

9. Kato S, Fukui K, Saigusa Y, Kubota K, Kodama S, Asahina $\mathrm{N}$, et al. Coronary flow reserve by cardiac magnetic 
resonance imaging in patients with diabetes mellitus. JACC Cardiovasc Imaging. 2019;12:2579-80. https://doi.org/10.1016/j. jcmg.2019.07.010.

10. Badawi A, Ryoo SG. Prevalence of comorbidities in the Middle East respiratory syndrome coronavirus (MERS-CoV): a systematic review and meta-analysis. Int J Infect Dis. 2016;49:129-33. https://doi.org/10.1016/j.ijid.2016.06.015.

11. Esler M, Kaye D, Thompson J, Jennings G, Cox H, Turner A, et al. Effects of aging on epinephrine secretion and regional release of epinephrine from the human heart. J Clin Endocrinol Metab. 1995;80:435-42. https://doi.org/10.1210/jcem.80.2.7852502.

12. Boisrame-Helms J, Kremer H, Schini-Kerth V, Meziani F. Endothelial dysfunction in sepsis. Curr Vasc Pharmacol. 2013;11:150-60.

13. Deng Q, Hu B, Zhang Y, Wang H, Zhou X, Hu W, et al. Suspected myocardial injury in patients with COVID-19: evidence from front-line clinical observation in Wuhan, China. Int J Cardiol. 2020. https://doi.org/10.1016/j.ijcard.2020.03.087.

14. Liu PP, Blet A, Smyth D, Li H. The science underlying COVID19: implications for the cardiovascular system. Circulation. 2020. https://doi.org/10.1161/CIRCULATIONAHA.120.047549.

15. Xu Z, Shi L, Wang Y, Zhang J, Huang L, Zhang C, et al. Pathological findings of COVID-19 associated with acute respiratory distress syndrome. Lancet Respir Med. 2020. https://doi.org/10.1016/ S2213-2600(20)30076-X.

16. Dolhnikoff M, Duarte-Neto AN, de Almeida Monteiro RA, Ferraz da Silva LF, Pierre de Oliveira E, Nascimento Saldiva PH, et al. Pathological evidence of pulmonary thrombotic phenomena in severe COVID-19. J Thromb Haemost. 2020. https://doi. org/10.1111/jth.14844.
17. Leonard-Lorant I, Delabranche X, Severac F, Helms J, Pauzet C, Collange O, et al. Acute pulmonary embolism in COVID-19 patients on CT angiography and relationship to D-dimer levels. Radiology. 2020. https://doi.org/10.1148/radiol.2020201561.

18. Walley KR, Becker CJ, Hogan RA, Teplinsky K, Wood LD. Progressive hypoxemia limits left ventricular oxygen consumption and contractility. Circ Res. 1988;63:849-59. https://doi. org/10.1161/01.res.63.5.849.

19. Wu J, Stefaniak J, Hafner C, Schramel JP, Kaun C, Wojta J, et al. Intermittent hypoxia causes inflammation and injury to human adult cardiac myocytes. Anesth Analg. 2016;122:373-80. https ://doi.org/10.1213/ANE.0000000000001048.

20. Kryzhanovskaya SY, Sazonova EN, Timoshin SS. Tissue homeostasis of the myocardium in newborn albino rats exposed to intrauterine hypoxia. Bull Exp Biol Med. 2004;138:23-5. https://doi. org/10.1023/b:bebm.0000046928.79653.10.

21. Walls AC, Park YJ, Tortorici MA, Wall A, McGuire AT, Veesler D. Structure, function, and antigenicity of the SARS-CoV-2 spike glycoprotein. Cell. 2020;181:281-92 e6. https://doi.org/10.1016/j. cell.2020.02.058.

22. Li L, Li L, Xiao L, Shangguan J. Progranulin ameliorates coxsackievirus-B3-induced viral myocarditis by downregulating Th1 and Th17 cells. Exp Cell Res. 2018;367:241-50. https://doi. org/10.1016/j.yexcr.2018.04.001.

23. Caforio ALP, Malipiero G, Marcolongo R, Iliceto S. Myocarditis: a clinical overview. Curr Cardiol Rep. 2017;19:63. https://doi. org/10.1007/s11886-017-0870-x. 